\title{
Meta
}

Journal des traducteurs

Translators' Journal

\section{Éloge de la traduction}

\section{Toru Shimizu}

Volume 33, numéro 1, mars 1988

Traduction et interprétation au Japon

Translation and Interpretation in Japan

URI : https://id.erudit.org/iderudit/004135ar

DOI : https://doi.org/10.7202/004135ar

Aller au sommaire du numéro

\section{Éditeur(s)}

Les Presses de l'Université de Montréal

\section{ISSN}

0026-0452 (imprimé)

1492-1421 (numérique)

Découvrir la revue

\section{Citer cet article}

Shimizu, T. (1988). Éloge de la traduction. Meta, 33(1), 104-107.

https://doi.org/10.7202/004135ar

\section{Résumé de l'article}

This article is the written text of a presentation made at a workshop on cooperation between authors and their translators at a symposium in Nice in November 1983 on the subject "French, a foreign language". Part of the author's ideas are based on his experience in translating the works of Michel Butor.

Language imposes a certain vision of the world on speakers, but not in a rigid and non-modifiable way. In this respect, translation has always played and continues to play an important role in shaping Japanese culture through experimentation with new styles and new approaches. In Japan, translation of literature is considered as participation in literary creation : most translations of literary and theoretical texts are done by academics, critics and writers, and the translated text of a book is generally followed by a rather long essay by the translator. In France, translators are generally not considered as literary personalities and their social status is accordingly low, in spite of a number of significant exceptions. And yet, translation forces one to innovate conceptually and linguistically, and therefore culturally; hence the positive nature of its contribution to the host culture, in particular through cooperation between authors and their translators.
Tous droits réservés @ Les Presses de l'Université de Montréal, 1988
Ce document est protégé par la loi sur le droit d'auteur. L'utilisation des services d’Érudit (y compris la reproduction) est assujettie à sa politique d'utilisation que vous pouvez consulter en ligne.

https://apropos.erudit.org/fr/usagers/politique-dutilisation/ 


\section{ÉLOGE DE LA TRADUCTION*}

TORU SHIMIZU

Meijigakuindaigaku, Tokyo, Japan

\section{SUMMARY}

This article is the written text of a presentation made at a workshop on cooperation between authors and their translators at a symposium in Nice in November 1983 on the subject "French, a foreign language". Part of the author's ideas are based on his experience in translating the works of Michel Butor.

Language imposes a certain vision of the world on speakers, but not in a rigid and non-modifiable way. In this respect, translation has always played and continues to play an important role in shaping Japanese culture through experimentation with new styles and new approaches. In Japan, translation of literature is considered as participation in literary creation: most translations of literary and theoretical texts are done by academics, critics and writers, and the translated text of a book is generally followed by a rather long essay by the translator. In France, translators are generally not considered as literary personalities and their social status is accordingly low, in spite of a number of significant exceptions. And yet, translation forces one to innovate conceptually and linguistically, and therefore culturally; hence the positive nature of its contribution to the host culture, in particular through cooperation between authors and their translators.

Cet atelier est consacré aux "problèmes de coopération entre l'auteur et ses traducteurs " et il serait de mon devoir de vous présenter des exemples de la coopération qui s'est établie avec Michel Butor, lors des traductions qu'il m'a été donné de faire de certaines de ses œuvres - depuis Kokorogawa / la Modification, qui a été publiée à Tokyo en 1959, jusqu'à la Littérature et la nuit / Bungaku to Yoru, un volume paru en 1982.

De nombreux souvenirs me reviennent en mémoire. Mais je voudrais me contenter de rappeler un épisode aussi particulier que significatif : il s'agit de la traduction de les Mots dans la peinture.

La première édition, publiée dans la collection illustrée "Sentiers de la création ", s'ouvre sur la reproduction d'un tableau de Marc Chagall, et se termine par celle d'une œuvre de George Segal. La reproduction de Chagall est imprimée au verso de la page de titre, c'est-à-dire sur la moitié gauche de la première double page, et celle de Segal est sur la moitié droite de la dernière double page. Ces tableaux sont peints tous deux en perspective, mais dans des directions opposées ; le point de fuite dans le tableau de Chagall, c'est-à-dire l'illustration sur la page gauche, se situe à droite de la page, tandis que dans celui de Segal, il est à gauche de la page. Si l'on ouvre donc ces deux pages en même temps, les deux perspectives se croisent juste au milieu du livre ; les deux points de fuite coïncident juste au milieu du volume.

Or, la première page des livres occidentaux est la dernière des livres japonais et vice versa. Si pour respecter l'ordre des pages, on met la reproduction de Chagall au début du livre japonais, et celle de Segal à la fin, on brisera la structure secrète de ce volume, parce que, dans ce cas, la perspective des deux illustrations diverge. Il n'y a plus 
stricte convergence des lignes de force. La fidélité superficielle du traducteur serait alors une trahison grave.

J'ai expliqué cet inconvénient à Butor — c'était lors du colloque de Cerisy —, et lui ai demandé la permission d'inverser l'ordre des deux reproductions. Butor m'a répondu en disant qu'il fallait absolument faire cette inversion, afin de garder tel quel ce qu'il avait imaginé comme une structure secrète du livre.

Je vous présente cet épisode, non pas pour prouver que la coopération entre l'auteur et son traducteur est utile, ce qui est l'évidence même, mais pour rappeler combien est grand l'écart entre le français et le japonais, et que ce décalage se vérifie aussi bien au niveau de la syntaxe que du lexique. La pensée court sur des rails tellement divergents que toute traduction doit, dès lors, être envisagée dans une problématique qui dépasse de loin les notions coutumières de "fidélité " ou d'" infidélité ".

En général, on discute des problèmes de la traduction en fonction des oppositions communément admises, "fidélité/infidélité ", " possibilité/impossibilité ". Le livre qu’a consacré Georges Mounin aux problèmes théoriques de la traduction est une trop rare exception. L'auteur veut que la discussion échappe au dogme de l'intraduisibilité, au fatalisme de l'échec, pour cerner clairement la marge de possibilité, et donc la liberté d'action dont dispose le traducteur. Dans ce livre, Mounin a accepté le fait qu'« une langue nous oblige à voir le monde d'une certaine manière, et nous empêche par conséquent de le voir d'autres manières ", et partant de cette idée, il soutient que, "bien que la traduction ne soit possible que dans une certaine mesure et dans certaines limites, il faut dans chaque cas déterminer cette mesure, décrire exactement ces limites, au lieu de les poser comme éternelles et absolues ». Voilà une attitude loyale, et il convient d'en reconnaître le bien-fondé. Pourtant, il me semble que Mounin considère la problématique de la traduction de façon statique. Il est vrai qu'une langue nous oblige à voir le monde d'une certaine manière, mais il n'est pas impossible de modifier cette perspective. Nous souhaitons que notre civilisation trouve en elle-même la force de se renouveler et, bien que les changements de l'expérience humaine ne se répercutent pas automatiquement dans la langue, nous pouvons travailler sur la langue pour donner un cours nouveau à la civilisation elle-même. Nous autres, traducteurs japonais, nous sommes enclins à nous résigner à l'impossibilité de toute traduction à cause du trop grand décalage linguistique entre la langue japonaise et les langues européennes, mais l'histoire de notre civilisation nous encourage en nous offrant des exemples des générations antérieures. Ceux qui nous ont précédé dans le temps, ont pu parfois modifier par leur travail même le système de la langue et faire évoluer notre civilisation.

Comme ce n'est pas ici un colloque sur la civilisation japonaise, je ne saurais entrer dans les détails. Mais, si je limite mon propos à la traduction proprement dite, on peut dire que, depuis près de cent ans, c'est-à-dire depuis que nous avons commencé à traduire, d'une manière quasi systématique, des œuvres littéraires et philosophiques européennes, la traduction se pratique chez nous comme l'expérimentation d'un nouveau style - la recherche d'un nouveau mode de pensée et d'une nouvelle rhétorique, la possibilité de concevoir de nouveaux domaines ou une nouvelle manière d'aborder des sujets connus...

Pour vous faire comprendre cette dynamique de la traduction dans la vie littéraire, je voudrais citer un exemple frappant. C'est le cas de la traduction d'un livre anglais, The Symbolist Movement in Literature d'Arthur Symons, par l'écrivain japonais IWANO Hōmei en 1913. Cette traduction est, si j'ose dire, la violence même. IWANO Hōmei a voulu être fidèle au style de Symons tant au niveau de la syntaxe que dans l'ordre du vocabulaire. Cette fidélité aussi rigoureuse que possible le conduisait à s'écarter 
des normes du "bon japonais" en employant des néologismes et une syntaxe d'une étrange violence.

C'était une traduction vraiment bizarre, mais en même temps bouleversante et féconde, car dès la génération suivante, un groupe de jeunes écrivains, poètes, critiques, universitaires, a été profondément marqué par ce texte. Parmi eux, le poète NAKAHARA Chūya (1907-1937), le critique KOBAYASHI Hideo (1907-1983), ou des universitaires comme SATO Maseaki (1905-1975).

Chose curieuse, ces jeunes intellectuels parlaient, pensaient, traduisaient et écrivaient en employant un vocabulaire et un style similaires. Dans ce laboratoire effervescent est né un nouveau style et ce groupe d'auteurs très jeunes a proposé de nouvelles orientations à la littérature de ce temps. Non seulement ils ont traduit Baudelaire, Verlaine, Mallarmé, Rimbaud, Valéry, Gide, Proust..., mais aussi ils ont rédigé des essais sur ces écrivains, et écrit leurs propres œuvres en y reprenant le vocabulaire et la nouvelle syntaxe forgés par IWANO Hōmei. C'était pendant les années 1920 et au début des années 1930.

Ce qui est essentiel dans leur cas, c'est qu'ils ont recherché à travers la lecture de textes étrangers et le travail de traduction une nouvelle conception de la littérature, et suscité une vraie renaissance dans l'histoire de la littérature japonaise.

Depuis lors, cette orientation se poursuit, et ce n'est pas un hasard si la façon de concevoir la traduction et de traiter le traducteur dans notre milieu littéraire diffêre tout à fait des habitudes qui prévalent en France. Au Japon, lorsqu'il s'agit de la traduction d'œuvres littéraires, philosophiques ou théoriques, le traducteur est presque toujours un universitaire, un critique littéraire ou un écrivain ; la couverture du livre traduit porte toujours le nom du traducteur, car il doit y figurer au même titre que celui de l'auteur. Et presque toujours, la traduction est suivie d'une étude ou d'un essai littéraire assez long rédigé par ce traducteur. Il y a bien sûr des traducteurs au sens où l'on entend ce mot en France. Mais leur domaine d'activité se limite aux livres de divertissement, comme les policiers de la série noire, ou la science-fiction, etc.

Je répète : au Japon, la traduction se définit comme une participation à la création littéraire. Si j'emprunte à Paul Valéry une définition de la littérature, elle est participation à « une exploration de tout un domaine de la sensibilité qui est gouverné par le langage ".

Je me demande s'il existe des orientations analogues en France. Il semble que non. En France, l'importance qui est reconnue à la traduction, le statut social du traducteur, sont beaucoup plus bas. La couverture du livre traduit ne porte pas toujours le nom du traducteur, ou il est donné en caractères minuscules. La mauvaise habitude de retraduire des livres japonais à partir de la version anglaise sévit toujours aujourd'hui. On n'exige, d'une traduction, que la lisibilité et la correction en français. On ne discute guère de la problématique de la traduction que dans le cadre des oppositions traditionnelles, "fidélité/infidélité ", "possibilité/impossibilité ".

Mais ce jugement est trop partiel. Je n'ignore pas l'existence de miracles, je n'ignore pas qu'il est des faiseurs de miracles, des saints patrons de la traduction française, depuis Amyot jusqu'à Valéry Larbaud. Si l'on cite quelques noms d'aujourd'hui, le travail parallèle de traduction et de réflexion qu'ont accompli Pierre Klosowski à propos de Nietzsche, Marthe Robert pour Kafka, est un très bon exemple dans ce domaine. Mais, en tant que traducteur dont la langue maternelle n'appartient pas à la famille indo-européenne, donc en tant que traducteur qui doit explorer de nouvelles possibilités dans sa langue propre, je suis particulièrement sensible au cas de Stéphane Mallarmé, qui, étant professeur d'anglais au lycée, a écrit les Mots anglais, Thèmes anglais, qui sont, je crois, une des meilleures initiations à l'étrangeté de son style, à la complexité des 
champs sémantiques où se déploie son vocabulaire. Autrement dit, l'invention du style et la formation du champ lexical chez Mallarmé sont largement dus à la lecture et à la traduction qu'il avait faites de textes anglais. On pourrait dire, mais ce serait exagérer un peu, que Mallarmé a écrit ses Divagations comme s'il traduisait en français ce qu'il pensait en anglais. Un écrivain et son propre traducteur ont collaboré dans la seule personne de Mallarmé.

En face du mot japonais 間 / ma, une notion esthétique spécifiquement japonaise, qui signifie une sorte d'espace actif, à la fois vacant et chargé d'une grande puissance, un traducteur français devrait tenter de trouver un équivalent en français, mais qui n'est jamais entré dans la critique littéraire française. Un traducteur japonais qui voudrait traduire Mallarmé devrait essayer d'inventer une nouvelle syntaxe qu'aucun poète japonais n'a jamais utilisée. Un texte qui aurait dû être écrit par un écrivain sera réalisé à travers la traduction; voilà l'idéal de la traduction. Et ces inventions pourraient peutêtre mettre en lumière quelque chose qui dort dans la couche profonde d'une langue et d'une civilisation.

J'avoue que tout ce que j'ai dit jusqu'ici a déjà été dit par Michel Butor dans son entretien avec Madeleine Santschi, intitulé Voyage avec Michel Butor. À propos d'un cri de terreur, qui est aussi une plaisanterie, poussé par un de ses traducteurs allemands, "Vous devriez quelquefois penser à nous. Aux difficultés que vous nous posez ", Butor répond comme suit :

Moi, au contraire, je travaille mes textes pour qu'ils soient aussi difficiles que possible à traduire dans une langue étrangère. Sinon, ce n'est même pas la peine de les traduire. C'est que les possibilités propres au français sont mal exploitées. Car, c'est justement en traduisant des textes difficiles qu'on va inventer des choses intéressantes en allemand. C'est pour cela qu'il vaut la peine de traduire.

Pour traduire ces " textes difficiles ", la coopération entre l'auteur et le traducteur est nécessaire, cela va de soi. Cette coopération doit être prise au sens élémentaire du not; entre l'un et l'autre doit s'instaurer le libre jeu des questions et des réponses. À travers cette coopération, le traducteur pourra, qui sait, inventer dans son texte traduit une valeur nouvelle, qui servira le commerce entre deux civilisations. Ce serait là la vraie coopération entre l'auteur et son traducteur.

Note

* Communication faite au colloque "Le français, langue étrangère ", Université de Nice, novembre 1983. 